\title{
Load Sensing Capability of Cementitious Matrixes- Nanomodified Cement Versus Carbon Nanotube Dispersion ${ }^{\dagger}$
}

\author{
Thanyarat Buasiri ${ }^{1, *}$, Karin Habermehl-Cwirzen ${ }^{1}$, Lukasz Krzeminski ${ }^{2}$ and Andrzej Cwirzen ${ }^{1}$ \\ 1 Building Materials, Department of Civil, Environmental and Natural Resources Engineering, Luleå \\ University of Technology, 97187 Luleå, Sweden; karin.habermehl-cwirzen@ltu.se (K.H.-C.); \\ andrzej.cwirzen@ltu.se (A.C.) \\ 2 The Institute of Engineering Materials and Biomaterials, Silesian University of Technology, 44-100 Gliwice, \\ Poland; lukasz.krzeminski@polsl.pl \\ * Correspondence: thanyarat.buasiri@ltu.se \\ + Presented at the 1st International Conference on Smart Materials for Sustainable Construction-SMASCO \\ 2019, Luleå, Sweden, 10-12 December 2019.
}

Published: 18 November 2019

\begin{abstract}
A cement-based matrix incorporating conductive materials such as carbon nanotubes and carbon nanofibers can have self-sensing capability. Both nanomaterials are characterized by excellent physical, mechanical and electrical properties. A disadvantage is that due to their hydrophobic nature it is very difficult to ensure uniform dispersion throughout the cementitious matrix. To overcome this problem a new nanomodified cement containing in-situ attached CNFs was developed leading to a very homogenous and conductive binder matrix. This study aimed to compare the piezoresistive responses of two types of matrixes, one based on the nanomodified cement and the second containing multi-walled carbon nanotubes. Several mortars were prepared containing either MWCNTs or the nanomodified cement, which partially replaced the untreated cement. The effective amount of the carbon nanomaterials was the same for both types of mixes and ranged from $0 \mathrm{wt.} \%$ to $0.271 \mathrm{wt} . \%$, calculated by the all binder weight. Changes in the electrical properties were determined while applying compressive load. The results showed that the binders based on the nanomodified cement have significantly better load sensing capabilities and are suitable for applications in monitoring systems.
\end{abstract}

Keywords: nanomodified cement; MWCNT; CNF; load sensing; sensing capability; cementitious matrixes 